\title{
EMPIRICAL ESTIMATE OF p-MODE FREQUENCY SHIFT FOR SOLAR CYCLE 23
}

\author{
Kiran Jain (kiran@uso.ernet.in), S. C. Tripathy \\ (sushant@uso.ernet.in), A. Bhatnagar (arvind@uso.ernet.in) \\ and Brajesh Kumar (brajesh@uso.ernet.in) \\ Udaipur Solar Observatory, A unit of Physical Research Laboratory, Off Bari \\ Road, Dewali, P. B. No. 198, Udaipur 313001, India
}

January 1, 2018

\begin{abstract}
.
We have obtained empirical relations between the p-mode frequency shift and the change in solar activity indices. The empirical relations are determined on the basis of frequencies obtained from BBSO and GONG stations during solar cycle 22. These relations are applied to estimate the change in mean frequency for the cycle 21 and 23. A remarkable agreement between the calculated and observed frequency shifts for the ascending phase of cycle 23, indicates that the derived relations are independent of epoch and do not change significantly from cycle to cycle. We propose that these relations could be used to estimate the shift in p-mode frequencies for past, present and future solar activity cycles, if the solar activity index is known. The maximum frequency shift for cycle 23 is estimated to be $265 \pm 90 \mathrm{nHz}$, corresponding to the predicted maximum smoothed sunspot number $118.1 \pm 35$.
\end{abstract}

\section{Introduction}

It is now well established that the solar p-mode oscillation frequencies vary with solar activity. The first evidence of this effect came from the analysis of low degree acoustic frequencies derived from solar irradiance data by the Active Cavity Radiometer instrument on board Solar Maximum Mission satellite (Woodard and Noyes, 1985). Using the Doppler velocity data, Pallé, Régulo, and Roca Cortés (1989) also showed that the frequency shifts are well correlated with solar activity cycle and obtained a shift of $0.44 \pm 0.06 \mu \mathrm{Hz}$ between the minimum and maximum of solar cycle 21. Later, several other authors have extended these studies to different epochs with new and improved data sets for intermediate (Woodard et al., 1991; Bachmann and Brown, 1993) and low degree modes (Jiménez-Reyes et al., 1998 and references therein). A consistent and continuous data set of intermediate degree p-mode frequencies is recently made available from the Global Oscillation Network Group (GONG) for the period May 1995 to October 1998, with an interval of 108 days. Using some of these data sets between August 1995 to August 1997, Bhatnagar, Jain, and Tripathy (1999) obtained a 
decrease of $0.06 \mu \mathrm{Hz}$ in the mean frequency during the descending phase of the solar cycle 22 and an increase of $0.04 \mu \mathrm{Hz}$ during the ascending phase of the cycle 23. Howe, Komm, and Hill (1999), using a subset of GONG frequencies, also demonstrated that the p-mode frequencies vary with solar activity cycle.

The prime motivation of this work is to derive empirical relations between the shift in frequency and change in the level of the activity indices. The derived relations could be used to estimate the frequency shift for past and future solar cycles. In this paper, we have used the change in activity indices corresponding to the same epoch as the frequency shifts instead of the actual value of activity. We find that the correlation between change in activity and frequency shifts is better as compared when the actual value of the activity indices were used. Using a similar approach Rhodes et al. (1993) showed that the frequency shifts between 1981 and 1989 are correlated with the change in various activity indices; e.g., the sunspot number, sunspot area, and irradiance measurements.

\section{Observational data}

The mode frequencies for cycle 22 in the intermediate degree range are available from the Big Bear Solar observatory (BBSO), LOWL instrument, South Pole expeditions and GONG project. As shown by Jain et al. (1999), frequencies derived from South Pole observations are systematically higher as compared with other three data sets. Similarly frequencies derived from LOWL instrument are from one year power spectra, which may not be valid for the study of short period solar cycle variation. Thus, in this study we use p-mode frequencies obtained from BBSO and GONG, for the period 1986 to 1996; these are summarised in Table I. It may be noted that we have used only the continuous and independent data sets from the GONG network. As it was shown by Libbrecht and Woodard (1990) that the mode frequency shift strongly depends on the frequencies, we have used only the common modes available between GONG and BBSO data sets. This selection criteria generated a total number of 412 common modes in the frequency range of 1500 and $3500 \mu \mathrm{Hz}$ and $\ell$ between 5 to 99 . Further, we have categorised the data sets into two groups according to the data source as shown in Table I. 
Table I. Frequency Data Sets

\begin{tabular}{llcc}
\hline Data Set & \multicolumn{1}{c}{ Epoch } & $\begin{array}{c}\text { Extent } \\
\text { (days) }\end{array}$ & $\begin{array}{c}\text { Number of Modes } \\
\text { (in } 0 \leq \ell \leq 100)\end{array}$ \\
\hline BBSO86 & Mar-Aug 1986 & 131 & 1095 \\
BBSO88 & Mar-Sep 1988 & 183 & 1095 \\
BBSO89 & Mar-Sep 1989 & 182 & 1095 \\
BBSO90 & Mar-Sep 1990 & 180 & 1095 \\
GM2 & 7 May-22 Aug 1995 & 108 & 1079 \\
GM5 & 23 Aug-8 Dec 1995 & 108 & 1078 \\
GM8 & 9 Dec-25 Mar 1996 & 108 & 1579 \\
GM11 & 26 Mar-11 July 1996 & 108 & 1143 \\
GM14 & 12 July-27 Oct 1996 & 108 & 1055 \\
\hline
\end{tabular}

\section{Analysis and results}

The mean frequency shift is calculated by taking simple difference between any two data sets chosen from the same group. With 9 frequency data sets (see Table I), the possible combinations (choosing any two at a time, out of the total data sets from an individual group) produced 16 values of the mean frequency shifts. In order to investigate how the frequency shifts are correlated with the change in the level of activity indices and to derive empirical relations between them, we have used seven different activity indices representing the magnetic and radiative indices. These indices are: $\mathrm{R}_{I}$, unsmoothed International sunspot number obtained from the Solar Geophysical Data (SGD); KPMI, Kitt Peak Magnetic Index from Kitt peak full disk magnetograms; MPSI, Magnetic Plage Strength Index from Mount Wilson magnetograms (Ulrich, 1991); FI, total flare index from SGD and Ataç (1999); He I, equivalent

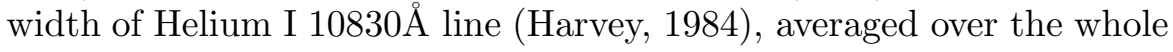
disk from Kitt peak; $\mathrm{F}_{10}$, integrated radio flux at $10.7 \mathrm{~cm}$ from SGD, and $\mathrm{R}_{s}$, smoothed International sunspot number obtained from SGD. A mean value for each activity index was computed, for the epoch corresponding to the actual frequency interval.

To study the relative variation in the mean frequency shift $\delta \nu$ with the change in activity index $\delta i$, we assume a linear relationship of the form:

$$
\delta \nu=a \delta i+b,
$$

where slope $a$ and intercept $b$ are obtained by performing a linear least square fit and is shown in Figure 1. The solid line represents the best 

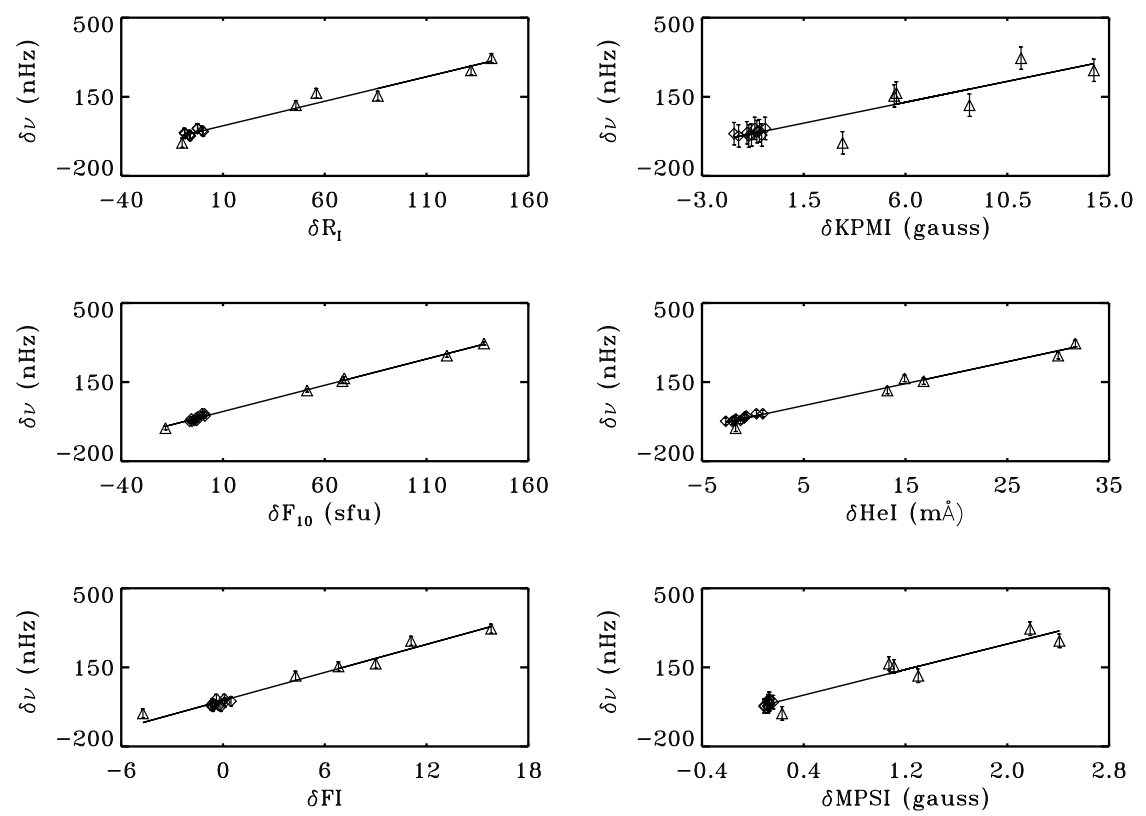

Figure 1. Linear regression of mean frequency shift with change in activity indices. The solid line is the least square fit. The triangles represent BBSO data while squares are for GONG data.

regression fit and confirms that the data sets are consistent with the assumption of a linear relationship. The bars represent $1 \sigma$ error in fitting.

The linear relationship given in Equation (1) is further tested by calculating the $\chi^{2}$, parametric Pearson's coefficient, $r_{P}$, the rank correlation coefficient, $r_{S}$, and their probabilities $P_{p}$ and $P_{s}$ respectively. Table II summarises the correlation statistics for all the data sets included in the fitting and shows that a positive correlation exists for all the activity indices. We further note that the best correlation is obtained for $\mathrm{F}_{10}$, which confirms earlier results (Bachmann and Brown, 1993; Bhatnagar, Jain, and Tripathy, 1999) that the radiative indices are better correlated with the frequency shifts. From the slope $a$ and the gradient $b$ obtained from the regression fitting, the following empirical relations between the shift, $\delta \nu$ and change in activity indices, $\delta i$ are formulated: 
EMPIRICAL ESTIMATE OF FREQUENCY SHIFT

Table II. Correlation statistics for Solar Cycle 22

\begin{tabular}{lccccc}
\hline Activity Index & $\chi^{2}$ & $r_{P}$ & $P_{p}$ & $r_{S}$ & $P_{s}$ \\
\hline $\mathrm{R}_{I}$ & 13 & 0.99 & $1.4 \mathrm{E}-12$ & 0.97 & $4.7 \mathrm{E}-10$ \\
KPMI & 72 & 0.91 & $7.1 \mathrm{E}-07$ & 0.73 & $1.2 \mathrm{E}-03$ \\
$\mathrm{~F}_{10}$ & 30 & 1.00 & $3.9 \mathrm{E}-19$ & 0.99 & $8.1 \mathrm{E}-13$ \\
He I & 12 & 0.99 & $3.2 \mathrm{E}-14$ & 0.93 & $1.9 \mathrm{E}-07$ \\
FI & 52 & 0.98 & $5.0 \mathrm{E}-12$ & 0.94 & $8.5 \mathrm{E}-08$ \\
MPSI & 25 & 0.97 & $3.7 \mathrm{E}-10$ & 0.81 & $1.4 \mathrm{E}-04$ \\
$\mathrm{R}_{s}$ & 38 & 0.99 & $1.0 \mathrm{E}-13$ & 0.95 & $1.4 \mathrm{E}-08$ \\
\hline
\end{tabular}

$$
\begin{aligned}
& \delta \nu=(2.44 \pm 0.18) \delta R_{I}-(6.00 \pm 1.36) \\
& \delta \nu=(18.70 \pm 1.62) \delta K P M I-(0.82 \pm 1.77) \\
& \delta \nu=(2.66 \pm 0.20) \delta F_{10}-(4.67 \pm 1.44) \\
& \delta \nu=(9.82 \pm 0.71) \delta H e I+(0.83 \pm 1.68) \\
& \delta \nu=(23.03 \pm 1.86) \delta F I-(11.10 \pm 1.22) \\
& \delta \nu=(159.01 \pm 11.90) \delta M P S I-(36.10 \pm 1.86) \\
& \delta \nu=(2.41 \pm 0.19) \delta R_{s}-(0.48 \pm 1.68)
\end{aligned}
$$

where $\delta \nu$ is given in $\mathrm{nHz}$ and the change in activity indices have their standard units. The second term in the parenthesis indicates $1 \sigma$ error. As mentioned previously, these relations are valid in the frequency range of 1500 to $3500 \mu \mathrm{Hz}$ and $\ell$ between 5 to 99 .

These relations are used to estimate the mean frequency shifts for solar cycle 21 through 23 by taking the frequency data set GM2 as the reference point. As an example, the estimated frequency shift obtained from Equation (8) for the smoothed sunspot number is plotted in Figure 2 and yields a shift of $0.37 \pm 0.03 \mu \mathrm{Hz}$ between the minimum and maximum of the solar cycle 21. This value is consistent with the earlier results by Pallé, Régulo, and Roca Cortés (1989) and Elsworth et al. (1990), who had obtained a shift of $0.44 \pm 0.06 \mu \mathrm{Hz}$ and $0.46 \pm$ $0.06 \mu \mathrm{Hz}$ respectively for the same period, using the Doppler velocity data of low degree $(\ell \leq 3)$ modes. From Figure 2, we notice that a very good agreement exists between the calculated values of $\delta \nu$ and the observed GONG frequencies (diamonds), however a small difference for BBSO (triangles) and LOWL (squares) is noticed. This difference may be interpreted as due to the use of different spectral lines in BBSO and 


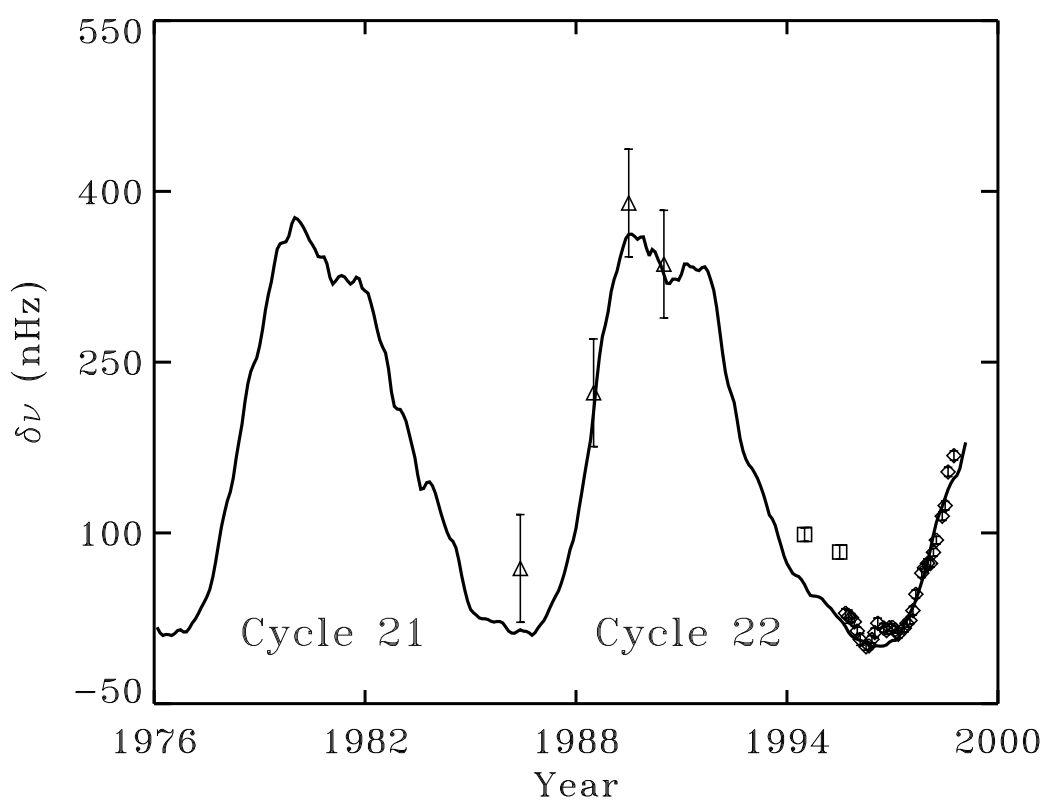

Figure 2. Comparison of estimated and observed mean frequency shift for cycle 21 through 23. The solid line is the mean shift as calculated from Equation (8). The observed shifts are represented by symbols; triangles for BBSO, squares for LOWL instrument and diamonds for GONG. The vertical line within the symbols represents the magnitude of $1 \sigma$ error in the frequncy shift.

LOWL instruments (Howe, Komm, and Hill, 1999) or different data reduction techniques.

\subsection{Comparison Between CyCle 22 And 23}

To investigate the validity of empirical relations for different cycles, we calculated the fitting parameters $a$ and $b$ for cycle 23, using the available GONG data in the frequency range of $1500-3500 \mu \mathrm{Hz}$ and $\ell$ between 5 to 99 . These parameters for solar cycle 22 and 23 are given in Table III and a reasonable agreeement is found for both the cycles.

Therefore, we propose that the derived linear relations are independent of solar cycle and can be used to estimate the frequency shifts for past, present and future solar cycles. This is illustrated in Figure 3, wherein we have plotted the calculated frequency shifts using Equations (2) and (4) and the observed frequency shifts for cycle 23. The dashed and solid lines represent the estimated $\delta \nu$, obtained from the relation for International sunspot number and $10.7 \mathrm{~cm}$ radio flux respectively, whereas the frequency shifts from the GONG data, with reference to 


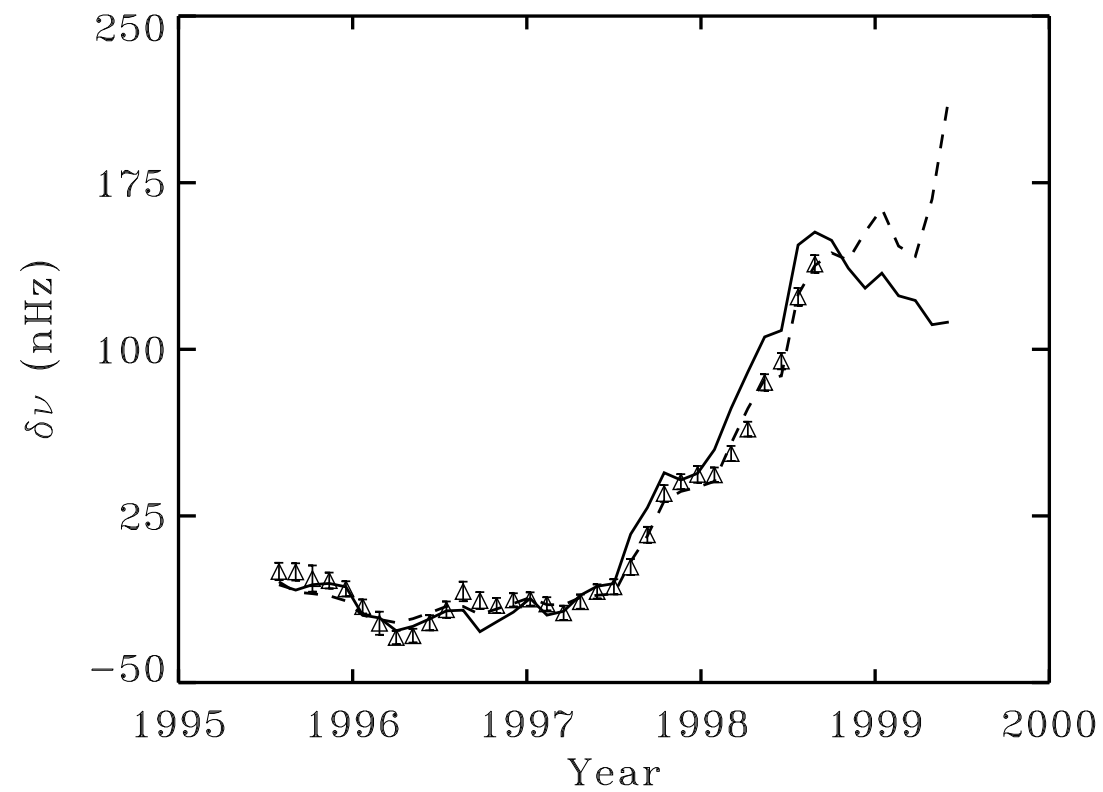

Figure 3. The estimated and observed frequency shifts for 1995-1998. The dashed line shows the shift, as estimated from the sunspot number (Equation (2)) and the solid line for the $10.7 \mathrm{~cm}$ (Equation (4)). The diamonds represent the observed frequency shifts from GONG data in the frequency range of 1500 and $3500 \mu \mathrm{Hz}$ and $\ell$ between 5 to 99 , where the mean shift is computed by taking GM2 as the reference.

Table III. Values of gradient $a$ (in $\mathrm{nHz} /$ activity) and intercept $b$ (in $\mathrm{nHz}$ ) obtained from least square fitting for solar cycle 22 and 23

\begin{tabular}{lrrrrr}
\hline \multirow{2}{*}{ Activity Index } & \multicolumn{2}{c}{ cycle 22} & & \multicolumn{2}{c}{ cycle 23} \\
\cline { 2 - 3 } \cline { 5 - 6 } & \multicolumn{1}{c}{$a$} & \multicolumn{1}{c}{$b$} & & \multicolumn{2}{c}{$a$} \\
\hline $\mathrm{R}_{I}$ & $2.44 \pm 0.18$ & $-6.00 \pm 1.36$ & & $2.01 \pm 0.07$ & $-4.20 \pm 0.56$ \\
KPMI & $18.70 \pm 1.62$ & $-0.82 \pm 1.77$ & & $22.6 \pm 0.79$ & $-1.86 \pm 0.56$ \\
F $_{10}$ & $2.66 \pm 0.20$ & $-4.67 \pm 1.44$ & & $2.72 \pm 0.09$ & $-3.25 \pm 0.56$ \\
HeI & $9.82 \pm 0.71$ & $+0.83 \pm 1.68$ & & $6.84 \pm 0.25$ & $+5.56 \pm 1.31$ \\
FI & $23.03 \pm 1.86$ & $-11.10 \pm 1.22$ & & $37.70 \pm 1.32$ & $-1.71 \pm 1.49$ \\
MPSI & $159.01 \pm 11.90$ & $-36.10 \pm 1.86$ & $107.00 \pm 4.17$ & $-15.12 \pm 2.06$ \\
& & & & \\
\hline
\end{tabular}



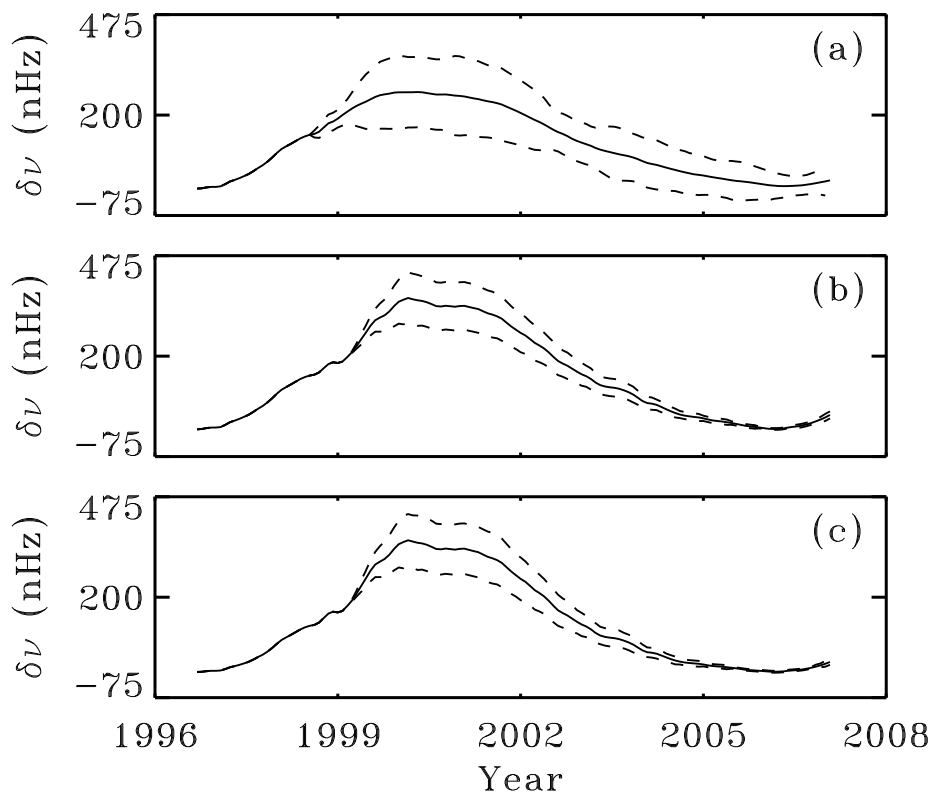

Figure 4. (a) Estimated $\delta \nu$ for cycle 23 from (a) the predicted smoothed sunspot number from SGD web site (solid line) (b) predicted smoothed sunspot number and (c) $10.7 \mathrm{~cm}$ radio flux from MSFC web site. The dashed lines show error in $\delta \nu$ due to the errors in predicted activity indices.

GM2, are shown as triangles. It is clear that the observed frequency shifts for cycle 23 are in close agreement with those obtained from the derived relations for cycle 22 .

In Figure 4, we have plotted the estimated frequency shifts for the current solar cycle 23, using predicted smoothed sunspot numbers and $10.7 \mathrm{~cm}$ flux. The solid line in Figure $4 a$ shows the predicted shift using $\mathrm{R}_{s}$, as listed in Solar Geophysical Data web page (http://www.ngdc.noaa/gov/stp/stp.html). The dashed lines are the predicted errors due to error in $\mathrm{R}_{s}$. Each smoothed sunspot number represents the average of two adjacent 12-month running mean of monthly means. The predicted value of sunspot number is based on the actual value of smoothed sunspot number for February 1999, which utilises the values of the averaged monthly means from August 1998 through August 1999. The predicted frequency shift for the solar cycle 23 is estimated to be $265 \pm 90 \mathrm{nHz}$ corresponding to the predicted maximum sunspot number of $118.1 \pm 35$. In Figure $4 b$ and $4 c$, we also show the estimated $\delta \nu$ for the current solar cycle using the predicted smoothed sunspot number and $10.7 \mathrm{~cm}$ radio flux obtained from http://wwwssl.msfc.nasa.gov/ssl/pad/solar/predict.htm. 
In summary, we have obtained empirical relations between the change in solar activity indices and the shift in p-mode frequencies for cycle 22. From these empirical relations, we have estimated p-mode frequency shift for the current cycle 23. It will be of interest to see how our predicted value agrees with the actual observed value during solar maximum in 2000. It is further shown that these relations are independent of the solar cycle and hence can be used to estimate the change in frequency for past, present and future epochs, if the solar activity index is known.

\section{Acknowledgements}

We thank T. Ataç, and R.K. Ulrich for supplying us the Flare index and MPSI data respectively. The BBSO p-mode data were acquired by Ken Libbrecht and Martin Woodard, Big Bear Solar Observatory, Caltech. LOWL data were obtained from (http://www.hao.ucar.edu/public/research/mlso/LowL/lowl.htm]), $\mathrm{NSO} /$ Kitt Peak magnetic, and Helium measurements used here are produced cooperatively by NSF/NOAO; NASA/GSFC and NOAA/SEL. This work utilizes data obtained by the Global Oscillation Network Group project, managed by the National Solar Observatory, a Division of the National Optical Astronomy Observatories, which is operated by AURA, Inc. under cooperative agreement with the National Science Foundation. The data were acquired by instruments operated by Big Bear Solar Observatory, High Altitude Observatory, Learmonth Solar Obsrvatory, Udaipur Solar Observatory, Instituto de Astrophsico de Canaris, and Cerro Tololo Interamerican Observatory. This work is partially supported under the CSIR Emeritus Scientist Scheme and Indo-US collaborative programme-NSF Grant INT-9710279.

\section{References}

Ataç, T.: 1999, private communication.

Bachmann, K. T. and Brown, T. M.: 1993, Astrophys. J. 411, L45.

Bhatnagar, A., Jain, K., and Tripathy, S. C.: 1999, Astrophys. J. 521, 885.

Elsworth, Y., Howe, R., Isaak, G. R., Mcleod, C. P., and New. R.: 1990, Nature 345, 322 .

Harvey, J. W.: 1984, in B. La Bonte, G. Chapman, H. Hudson, and R. C. Wilson (eds.), Workshop on Solar Irradiance Variations on Active Region Time Scales, NASA CP-2310; NASA, Washington, p. 197.

Howe, R., Komm, R., and Hill, F.: 1999, Astrophys. J. 524, 1084.

Jain, K., Tripathy, S. C., Kumar, B., and Bhatnagar, A. 1999, Bull. Astron. Soc. India (in Press). 
Jiménez-Reyes, S. J., Régulo, C , Pallé, P. L., and Roca Cortés, T.: 1998, Astron. Astrophys. 329, 1119.

Libbrecht, K. G., and Woodard, M. F.: 1990, in Osaki, Y. and Shibahashi, H. (eds.), Progress of Seismology of the Sun and Stars, Springer, p. 145.

Pallé. P. L., Régulo, C., and Roca Cortés, T.: 1989, Astron. Astrophys. 224, 253.

Rhodes, E. J., Jr., Cacciani, A., Korzennik, S. G., and Ulrich, R. K.: 1993, Astrophys. J. 406, 714 .

Ulrich, R. K.: 1991, Adv. Space Res. 11(4), 217.

Woodard, M. F., Kuhn, J. R., Murray, N., and Libbrecht, K. G.: 1991, Astrophys. J. 373, L81.

Woodard, M. F. and Noyes, R. W.: 1985, Nature 318, 449.

Address for Offprints: Dr. Kiran Jain

Udaipur Solar Observatory

Off Bari Road, Dewali, P. B. No. 198,

Udaipur - 313001,

India

e-mail:kiran@uso.ernet.in 